\title{
ALTERNATION THEOREM FOR $C(I, X)$ AND APPLICATION TO BEST LOCAL APPROXIMATION
}

\author{
A. AL-ZAMEL AND R. KHALIL
}

\begin{abstract}
Let $X$ be a Banach space with the approximation property, and $C(I, X)$ the space of continuous functions defined on $I=[0,1]$ with values in $X$. Let $u_{i} \in C(I, X), i=1,2, \cdots, n$ and $M=\operatorname{span}\left\{u_{1}, \ldots\right.$, $\left.u_{n}\right\}$. The object of this paper is to prove that if $\left\{u_{1}, \ldots, u_{n}\right\}$ satisfies certain conditions, then for $f \in C(I, X)$ and $g \in M$ we have $\|f-g\|=$ $\inf \{\|f-h\|: h \in M\}$ if and only if $f-g$ has at least $n$-zeros. An application to best local approximation in $C(I, X)$ is given.
\end{abstract}

\section{Introduction}

Let $I=[0,1]$ and $C(I)$ the space of real valued continous functions. If $\left\{u_{1}, \ldots, u_{n}\right\}$ forms a $T$-system in $C(I)$, then the space $M=\operatorname{span}\left\{u_{1}, \ldots, u_{n}\right\}$ is a Chebechev subspace of $C(I),[3$ p.81]. Hence for each $f \in C(I)$ there exists a unique $g \in M$ such that

$$
\|f-g\|=d(f, M)=\inf \{\|f-h\|: h \in M\} .
$$

The Alternation Theorem, [3, p.75], gives an important simple characterization of $g:\|f-g\|=d(f, M)$ if and only if $f-g$ has at least $n$-zeros".

Chui, Shisha and Smith [4] used the Alternation Theorem to prove the existence of what they called "best local approximation" in $C(I)$.

The object of this paper is to study the Alternation Theorem and the problem of best local approximation in vector valued function spaces. It turns out

\footnotetext{
Received October 15, 1991; revised November 18, 1992.
} 
that if we assume that the Banach space $X$ has the so-called approximation property, and the set $\left\{u_{1}, \ldots, u_{n}\right\} \subset C(I, X)$ satisfies certain conditions, then one has an Alternation Theorem, which then applied to prove results on best local approximation in $C(I, X)$.

Throughtout this paper, if $X$ is a Banach space, then $X^{*}$ is the dual of $X$, and for $x \in X$, and $x^{*} \in X^{*}$ we write $\left\langle x, x^{*}\right\rangle$ for $x^{*}(x)$. The unit ball of $X^{*}$ is denoted by $B_{1}\left(X^{*}\right)$. The unit mass measure at $a \in I$ is denoted by $\delta_{a}$. Hence $\left\langle f, \delta_{a}\right\rangle=f(a)$. The set of reals is denoted by $R$.

\section{1. $C(I, X)$ As Scalar Functions}

For Banach spaces $X$ and $Y$, let $X \stackrel{\vee}{\oplus}(X \hat{\oplus} Y)$ denote the injective (projective) tensor product of $X$ with $Y$, [5. Chap 1]. It is well known that $C(I, X)$ is isometrically isomorphic to $C(I) \stackrel{\oplus}{\oplus},[5 ; \mathrm{p} .9]$ for any Banach space $X$. In general it is not ture that $(X \hat{\oplus} Y)^{*}=X^{*} \stackrel{\vee}{\oplus} Y^{*}$. In this work, we will choose $X$ such that $(C(I) \stackrel{\ominus}{\oplus} X)^{*}=[C(I)]^{*} \hat{\oplus} X^{*}$. Banach spaces with the so-called approximation property satisfies such equality, [5 Chap. 1]. Further, $L^{p}(\mu), 1 \leq p \leq \infty$ and $C(I)$ have the approximation property, [2, p. 245].

Every $t \in I$ represents the unit mass measure $\delta_{t}$. Hence $I \subset M(I)=C(I)^{*}$, the space of Borel measures on $I$. Further $I$ is compact in $M(I)$ with the $w^{*}$ topology. We also have $B_{1}\left(X^{*}\right)$ is compact with the $w^{*}$-topology, by the Alaoglu Theorem. Hence $I \times B_{1}\left(X^{*}\right)$ is a compact space in the product topology.

Now every $f \in C(I, X)$ can be considered as a continuous function defined on $B_{1}\left([C(I, X)]^{*}\right)=B_{1}\left(M(I) \hat{\oplus} X^{*}\right)$ (Since $X$ is assumed to have the approximation property). Since $I \times B_{1}\left(X^{*}\right)$ is closed in $[C(I, X)]^{*}$ and $I \times B_{1}\left(X^{*}\right) \subset$ $B_{1}\left(M(I) \hat{\oplus} X^{*}\right)$, we get $I \times B_{1}\left(X^{*}\right)$ is closed in $B_{1}\left(M(I) \hat{\oplus} X^{*}\right)$. Here, the topology we refere to is the $w^{*}$-topology.

Finally, since for $f \in C(I, X)$ we have 


$$
\begin{aligned}
\|f\| & =\sup _{t \in I}\|f(t)\| \\
& =\sup _{t \in I} \sup _{x^{*} \in B_{1}\left(X^{*}\right)}\left|\left\langle f(t), x^{*}\right\rangle\right| \\
& =\sup _{\delta_{t} \oplus x^{*}}\left|\left\langle f, \delta_{t} \oplus x^{*}\right\rangle\right|,
\end{aligned}
$$

we can consider, and we will, $f: I \times B_{1}\left(X^{*}\right) \rightarrow R$.

\section{Vector Valued Alternation Theorem}

Let $f \in C(I, X)$. We set

$$
m(f)=\left\{\delta_{t} \oplus x^{*}:\left\langle f, \delta_{t} \oplus x^{*}\right\rangle=\|f\|, \quad t \in I, x^{*} \in B_{1}\left(X^{*}\right)\right\} .
$$

Then one can easily prove:

Lemma 2.1. $m(f)$ is compact in $I \times B_{1}\left(X^{*}\right)$.

Now, let $\left\{u_{1}, \ldots, u_{n}\right\} \subset C(I, X)$. For $\left(t, x^{*}\right) \in I \times B_{1}\left(X^{*}\right)$ we set

$$
\hat{u}\left(t, x^{*}\right)=\left(\left\langle u_{1}(t), x^{*}>, \cdots,<u_{n}(t), x^{*}\right\rangle\right) .
$$

Thus $\hat{u}\left(t, x^{*}\right) \in R^{n}$ for each $\left(t, x^{*}\right) \in I \times B_{1}\left(X^{*}\right)$. Then

Lemma 2.2. Let $f \in C(I, X)$. Then the set

$$
E=\left\{\left\langle f(t), x^{*}\right\rangle \hat{u}\left(t, x^{*}\right):\left(t, x^{*}\right) \in m(f)\right\}
$$

is a compact set in $R^{n}$.

Proof. consider the function

$$
\begin{aligned}
& \psi: I \times B_{1}\left(x^{*}\right) \rightarrow R^{n} \\
& \psi\left(t, x^{*}\right)=\left\langle f(t), x^{*}\right\rangle \hat{u}\left(t, x^{*}\right) .
\end{aligned}
$$

Since $f, u_{1}, \ldots, u_{n}$ are continuous functions, then $\psi$ is continuous. But

$$
E=\left\{\psi\left(t, x^{*}\right):\left(t, x^{*}\right) \in m(f)\right\}
$$


But by Lemma 2.1, $m(f)$ is compact. Hence $E$ is compact.

Now, let $M$ be subspace of $C(I, X)$ generated by $u_{1}, \ldots, u_{n}$. Hence if $g \in$ $M$, then $g=\sum_{i=1}^{n} a_{i} u_{i}, a_{i} \in R$. Since $M$ is finite dimensional, then for each $f \in C(I, X)$ there exists at least one $g \in M$ such that

$$
\|f-g\|=d(f, M)=\inf \{\|f-h\|: h \in M\} .
$$

Now we prove the Characterization Theorem $[3, \mathrm{p} .73]$ for the space $C(I, X)$

Theorem 2.3. Let $f \in C(I, X)$ and $g \in M$. The following are equivalent:

(i) $\|f-g\|=d(f, M)$

(ii) $\underline{O}=(0, \cdots, 0)$ is in the convex hull of $E=\left\{\left(r\left(t, x^{*}\right) \hat{u}\left(t, x^{*}\right) ;\left(t, x^{*}\right) \in m(r)\right\}\right.$ in $R^{n}$, where $r\left(t, x^{*}\right)=\left\langle f(t)-g(t), x^{*}\right\rangle$.

Proof. (ii) $\rightarrow$ (i). Let $r\left(t, x^{*}\right)=\left\langle f(t)-g(t), x^{*}\right\rangle$. If possible assume that $g$ is not a best approximant to $f$ in $M$. Hence there exists $h \in M$ such that $\|r-h\|<\|r\|$. Consequently,

$$
\| r\left(t, x^{*}\right)-\left\langle h(t), x^{*}\right\rangle|<| r\left(t, x^{*}\right) \mid
$$

for all $\left(t, x^{*}\right) \in m(r)$. Equation (1) implies that

$$
r\left(t, x^{*}\right)\left\langle h(t), x^{*}\right\rangle>0
$$

for all $\left(t, x^{*}\right) \in m(r)$.

Since $h \in M$, then $h=\sum_{i=1}^{n} b_{i} u_{i}$, for some $b_{i} \in R, i=1, \cdots, n$. Hence

$$
\left\langle h(t), x^{*}\right\rangle=\sum_{i=1}^{n} b_{i}\left\langle u_{i}(t), x^{*}\right\rangle=\left\langle b, \hat{u}\left(t, x^{*}\right)\right\rangle,
$$

where $b=\left(b_{1}, \ldots, b_{n}\right) \in R^{n}$. Hence, Equation (2) implies

$$
r\left(t, x^{*}\right)\left\langle b, \hat{u}\left(t, x^{*}\right)\right\rangle>0
$$


for all $\left(t, x^{*}\right) \in m(r)$. But $r\left(t, x^{*}\right)=\|r\|>0$ for all $\left(t, x^{*}\right) \in m(r)$. Hence $\left\langle b, \hat{u}\left(t, x^{*}\right)\right\rangle>0$ for all $\left(t, x^{*}\right) \in m(r)$. By Lemma 2.2 , the set $E=\left\{r\left(t, x^{*}\right) \hat{u}(t\right.$, $\left.\left.x^{*}\right):\left(t, x^{*}\right) \in m(r)\right\}$ is compact in $R^{n}$. Hence, [3, p.19], we get

$$
\underline{O}=(0,0, \cdots, 0) \notin \text { Convexhull of } E \text {. }
$$

Conversely。 (i) $\rightarrow$ (ii). Let the vector $\underline{O}=(0, \cdots, 0) \notin$ convexhull of $E=$ $\left\{r\left(t, x^{*}\right) \hat{u}\left(t, x^{*}\right):\left(t, x^{*}\right) \in m(r)\right\}$. Hence, [3, p.19], there exists $b \in R^{n}$ such that $\left\langle b, r\left(t, x^{*}\right) \hat{u}\left(t, x^{*}\right)\right\rangle>0$ for all $\left(t, x^{*}\right) \in m(r)$. By Lemma 2.1, $m(r)$ is compact. Hence, there exists $\mathcal{E}>0$ and $\left(t_{0}, x_{0}^{*}\right)$ such that

$$
\begin{aligned}
\mathcal{E} & =\inf \left\{r\left(t, x^{*}\right)\left\langle b, \hat{u}\left(t, x^{*}\right)\right\rangle:\left(t, x^{*}\right) \in m(r)\right\} \\
& =r\left(t_{0}, x_{0}^{*}\right)\left\langle b, \hat{u}\left(t_{0}, x_{0}^{*}\right)\right\rangle .
\end{aligned}
$$

Now, let

$$
K_{1}=\left\{\left(t, x^{*}\right) \in I \times B_{1}\left(X^{*}\right): r\left(t, x^{*}\right)<b, \hat{u}\left(t, x^{*}\right) \leq \mathcal{E} / 2\right\}
$$

The set $K_{1}$ is closed (and hence compact) in $I \times B_{1}\left(X^{*}\right)$. Further $K_{1} \cap m(r)=\phi$. Since $r$ is continuous, and $K_{1}$ is compact, then $|r|$ attains its maximum on $K_{1}$ and if

$$
\alpha=\max \left\{\left|r\left(t, x^{*}\right)\right|:\left(t, x^{*}\right) \in K_{1}\right\},
$$

then $\alpha<\|r\|$. Now choose $\lambda>0$ such that $0<\lambda<\frac{\|r\|-\alpha}{\left\|\sum_{i=1}^{n} b_{i} u_{i}\right\|}$. Hence for any $\left(t, x^{*}\right) \in K$, we have

$$
\begin{aligned}
\left|r\left(t, x^{*}\right)-\lambda \sum_{i=1}^{n} b_{i}\left\langle u_{1}(t), x^{*}\right\rangle\right| & \leq\left|r\left(t, x^{*}\right)\right|+\lambda\left|\sum_{i=1}^{n} b_{i}\left\langle u_{i}(t), x^{*}\right\rangle\right| \\
& \leq \alpha+\lambda\left\|\sum_{i=1}^{n} b_{i} u_{i}\right\| \\
& <\|r\| \quad\left(\text { since } \lambda<\frac{\|r\|-\alpha}{\left\|\sum_{i=1}^{n} b_{i} u_{i}\right\|}\right)
\end{aligned}
$$


On the other hand, if $\left(t, x^{*}\right) \notin K_{1}$, then we can choose $\lambda$ to satisfy the addifional condition $0<\lambda<\frac{\mathcal{E}}{\left\|\sum_{i=1}^{n} b_{i} u_{i}\right\|^{2}}$. Since $r\left(t, x^{*}\right)\left\langle b, \hat{u}\left(t, x^{*}\right)\right\rangle>\mathcal{E} / 2$ for all $\left(t, x^{*}\right) \notin K_{1}$, we get:

$$
\begin{aligned}
\left|r\left(t, x^{*}\right)-\lambda \Sigma b_{i} u_{i}\left(t, x^{*}\right)\right|^{2} & \leq\|r\|^{2}+\lambda\left\|\Sigma b_{i} u_{i}\left(t, x^{*}\right)\right\|^{2}-\mathcal{E} \\
& <\|r\|^{2} \quad\left(\text { since } \lambda<\frac{\mathcal{E}}{\left\|\Sigma b_{i} u_{i}\right\|^{2}}\right)
\end{aligned}
$$

Equations $\left(^{*}\right)$ and $\left({ }^{* *}\right)$ implies that $g$ is not a best approximant of $f$ in $M$. This ends the proof.

For $f \in C(I, X)$ and $x^{*} \in B_{1}\left(X^{*}\right)$ set:

$$
m\left(f, x^{*}\right)=\left\{\left(t, x^{*}\right):\left|\left\langle f(t), x^{*}\right\rangle\right|=\|f\|\right\} .
$$

clearly $m\left(f, x^{*}\right)$ is a closed subset of $m(f)$. Then Theorem 2.3 is valid in the following setting.

Theorem 2.4. Let $f \in C(I, X)$ and $g \in M$. The following are equivalent:

(i) $\|f-g\|=d(f, M)$

(ii) $\underline{Q}=(0,0, \cdots, 0)$ is in the convex hull of $E\left(x^{*}\right)=\left\{r\left(t, x^{*}\right) \hat{u}\left(t, x^{*}\right):\left(t, x^{*}\right) \in\right.$ $\left.m\left(f-g, x^{*}\right)\right\}$.

We need one more result before we can prove the Alternation Theorem.

Set

$$
N=\left\{x^{*} \in B_{1}\left(X^{*}\right): \hat{u}\left(t, x^{*}\right) \neq \underline{0} \text { for all } t \in I\right\} .
$$

For general $u_{1}, \ldots, u_{n}$ in $C(I, X)$, the set $N$ could be empty. This occures if $u_{1}, \ldots, u_{n}$ have a common zero.

Definition 2.5. The set $\left\{u_{1}, \ldots, u_{n}\right\} \subset C(I, X)$ is said to satisfy the Haar condition if there exists at least one $x^{*} \in N$ such that

$$
D\left(t_{1}, \ldots, t_{n}, x^{*}\right)=\left|\begin{array}{c}
\left\langle u_{1}\left(t_{1}\right), x^{*}\right\rangle \cdots\left\langle u_{1}\left(t_{n}\right), x^{*}\right\rangle \\
\left\langle u_{n}\left(t_{1}\right), x^{*}\right\rangle \cdots\left\langle u_{n}\left(t_{n}\right), x^{*}\right\rangle
\end{array}\right| \neq 0
$$

for all $t_{1}<t_{2}<\ldots<t_{n}$ in $I$. Set $N(D)=\left\{x^{*} \in N: D\left(t_{1}, \ldots, t_{n}, x^{*}\right) \neq 0\right.$ for all $t_{1}<t_{2}<\ldots<t_{n}$ in $\left.I\right\}$. 
A simple example satisfying the Haar condition is:

$$
u_{1}=t \oplus x, \cdots, u_{n}=t^{n} \oplus x,
$$

where $x \neq 0$ is in the Banach space $X$.

The set $I^{n} \times\left\{x^{*}\right\}=\left\{\left(t_{1}, \ldots, t_{n}, x^{*}\right): t_{i} \in I\right\}$ is a convex subset of $I^{n} \times$ $B_{1}\left(X^{*}\right)$. Hence the continuouity of $D\left(t_{1}, \ldots, t_{n}, x^{*}\right)$ as a real valued function on $I^{n} \times B_{1}\left(X^{*}\right)$ implies (using the Intermediate Value Theorem) that $D$ has the same sign on $I^{n} \times\left\{x^{*}\right\}$. Then, togother with Lemma 1 [p. 74] we get:

Lemma 2.6. Let $x^{*} \in N(D)$ and $\left\{t_{0}, \ldots, t_{n}\right\}$ be a set of $n+1$ distinct elements in $I$, and $\lambda_{0}, \ldots, \lambda_{n}$ be non-zero real numbers. Let $E\left(x^{*}\right)=$ $\left\{\lambda_{0} \hat{u}\left(t_{0}, x^{*}\right), \ldots, \lambda_{n} \hat{u}\left(t_{n}, x^{*}\right)\right\}$. The $\underline{O}=(0, \cdots, 0) \in$ convexhull of $E\left(x^{*}\right)$ if and only if $\lambda_{i} \lambda_{i-1}<0$ for $i=1, \cdots n$.

Now we prove:

Theorem 2.7. (Alternation Theorem). Let $\left\{u_{1}, \ldots, u_{n}\right\} \subset C(I, X)$ satisfy the Haar condition. Let $f \in C(I, X)$ and $g \in M$. The following are Equivalent:

(i) $\|f-g\|=d(f, M)$

(ii) $f-g$ has at least $n$-zeros.

Proof. Let $r=f-g$. By Theorem 2.3, (i) is satisfied if and only if $\underline{Q}=(0, \cdots, 0) \in$ convexhull of $E\left(x^{*}\right)=\left\{r\left(t, x^{*}\right) \hat{u}\left(t, x^{*}\right):\left(t, x^{*}\right) \in m(r)\right\}$. Since $E\left(x^{*}\right)$ is compact, then every point in the convexhull of $E\left(x^{*}\right)$ is a convex linear combination of at most $n$-elements of $E\left(x^{*}\right)$. Thus there exists $\lambda_{0}, \ldots, \lambda_{k} \in(0,1)$ such that $\sum_{i=0}^{k} \lambda_{i}=1$ and $0=\sum_{i=0}^{k} \lambda_{i} r\left(t_{i}, x^{*}\right) \hat{u}\left(t_{i}, x^{*}\right)$. By Caratheodory Theorem [3 p.17], we have $k \leq n$.

Since the set $\left\{u_{1}, \ldots, u_{n}\right\}$ satisfy the Haar condition, the elements of any subset $\left\{\hat{u}\left(t_{i_{k}}, x^{*}\right): 1 \leq k \leq n\right\}$ is independent in $R^{n}$. Consequently $k \geq n$. Hence $k=n$.

Lemma 2.6 now implies that $\lambda_{i} r\left(t_{i}, x^{*}\right)$ has at least $n+1$ alternation. But $\lambda_{i}>0$. Thus $r$ has at least $n$-zeros. This ends the proof. 


\section{Best Local approximation}

Let $\left\{u_{1}, \ldots, u_{n}\right\} \subset C(I, X)$ and $M=\operatorname{span}\left\{u_{1}, \ldots, u_{n}\right\}$. For $\mathcal{E} \in(0,1)$ let $I_{\mathcal{E}}=[0, \mathcal{E}]$ and $M_{\mathcal{E}}=\left.M\right|_{I_{\mathcal{E}}}$, the restriction of $M$ to $I_{\mathcal{E}}$. Then $M_{\mathcal{E}} \subset C\left(I_{\mathcal{E}}, X\right)$. Let $f \in C(I, X)$ and $f_{\mathcal{E}}=\left.f\right|_{I_{\mathcal{E}}}$. Since $M_{\mathcal{E}}$ is finite dimensional for all $\mathcal{E}$, it follows that for each $\mathcal{E}$ there exists $P_{\mathcal{E}}(f) \in M_{\mathcal{E}}$ such that

$$
\left\|f_{\mathcal{E}}-P_{\mathcal{E}}(f)\right\|=d\left(f_{\mathcal{E}}, M_{\mathcal{E}}\right) .
$$

The net $\left(P_{\mathcal{E}}(f)\right)$ need not to converge as $\mathcal{E} \rightarrow 0^{+}$. Following Chui, Shisha and Smith [4], "if $\left(P_{\mathcal{E}}(f)\right)$ converges uniformly on some interval $\left[0, \mathcal{E}_{0}\right]$ to some $P_{0}(f) \in M$, then we say that $P_{0}(f)$ is a best local approximation of $f . "$

The object of this section, is to use the results in section II of this paper to prove a similar type Theorems of Chui-etal [4, Theorem 2.1] for vector valued continous functions, with the uniform norm and with the $L^{1}$-norm.

For $f \in C(I, X)$ we say that $f$ is weakly differentiable on $I$ if for each $t \in(0,1)$

$$
\lim _{\mathcal{E} \rightarrow 0}\left\langle\frac{f(t+\mathcal{E})-f(t)}{\mathcal{E}}, x^{*}\right\rangle
$$

exists for each $x^{*} \in X^{*}$. We will write $f^{\prime}\left(t, x^{*}\right)$ for such limit. Let $C_{\omega}^{n}(I, X)$ denote the space of $n$-times weakly differnetiable functions. We let $f^{(j)}\left(t, x^{*}\right)$ denote the $j^{\text {th }}$-derevative associated with $t$ and $x^{*}$.

Now we assume that the set $\left\{u_{1}, \ldots, u_{n}\right\} \subset C_{\omega}^{n}(I, X)$. For $x^{*} \in B_{1}\left(X^{*}\right)$, we let

$$
A_{n}\left(x^{*}\right)=\left|\begin{array}{cc}
\left\langle u_{1}(0), x^{*}\right\rangle & \cdots,\left\langle u_{n}(0), x^{*}\right\rangle \\
\vdots & \vdots \\
u_{1}^{(n-1)}\left(0, x^{*}\right) \cdots u_{n}^{(n-1)}\left(0, x^{*}\right)
\end{array}\right|
$$

Now we prove

Theorem 3.1. Let $\left\{u_{1}, \ldots, u_{n}\right\} \subset C_{\omega}^{n}(I, X)$ satisfy the Haar Condition. Assume that for every $f \in C_{\omega}^{n}(I, X)$ the net $P_{\mathcal{E}}(f)$ converges uniformely to $f_{0}$ as $\mathcal{E} \rightarrow 0^{+}$. Then the matrix $A_{n}\left(x^{*}\right)$ is non-singular for every $x^{*} \neq 0$ in $B_{1}\left(X^{*}\right)$. 
Proof. Since $P_{\mathcal{E}}(f) \in M$ for each $\mathcal{E}>0$, it follows that $P_{0}(f) \in M$. Further, since

$$
\left\|P_{\mathcal{E}}(f)-f_{\mathcal{E}}\right\|=d\left(f_{\mathcal{E}}, M_{\mathcal{E}}\right)
$$

then by Theorem 2.7 there exists $\left(t_{i}(\mathcal{E})\right)_{i=1}^{n}$, such that $0<t_{1}(\mathcal{E})<t_{2}(\mathcal{E})<\cdots<$ $t_{n}(\mathcal{E})<\mathcal{E}$ and

$$
P_{\mathcal{E}}(f)\left(t_{i}(\mathcal{E})\right)-f\left(t_{i}(\mathcal{E})\right)=0, \quad\left(f_{\mathcal{E}}\left(t_{i}(\mathcal{E})\right)=f\left(t_{i}(\mathcal{E})\right)\right.
$$

Thus by Rolle's Theorem, for each $x^{*} \in$ in $B_{1}\left(X^{*}\right)$ there exists $\left(s_{j}(\mathcal{E})\right)_{j=1}^{n}$ such that

$$
P_{\mathcal{E}}(f)^{j-1}\left(s_{j}(\mathcal{E}), x^{*}\right)-f^{j-1}\left(s_{j}(\mathcal{E}), x^{*}\right)=0
$$

where $0<S_{1}(\mathcal{E})<\cdots<S_{n-j+1}(\mathcal{E})<\mathcal{E}$. Now fixing $x^{*}$ and taking the limit as $\mathcal{E} \rightarrow 0^{+}$in $(1)$ we get

$$
\lim _{\mathcal{E} \rightarrow 0^{+}} P_{\mathcal{E}}(f)^{j-1}\left(s_{j}(\mathcal{E}), x^{*}\right)=f^{(j-1)}\left(0, x^{*}\right),
$$

$j=1, \cdots, n$, and (2) holds for all $f \in C_{\omega}^{n}(I, X)$ and $x^{*} \in B_{1}\left(X^{*}\right)$.

Now, since $P_{\mathcal{E}}(f) \in M$, we have

$$
P_{\mathcal{E}}(f)(t)=\sum_{i=1}^{n} a_{i}(\mathcal{E}, f) u_{i}(t) .
$$

Further, that $P_{\mathcal{E}}(f) \underset{\mathcal{E}}{\longrightarrow} P_{0}(f)$ and that $M$ is finite dimensional implies that $a_{i}(\mathcal{E}, f) \underset{\mathcal{E}}{\longrightarrow} a_{i}(f)$ say for each $i=1, \cdots, n$. Hence from Equation (2) and the fact that $u_{i} \in C_{\omega}^{n}(I, X)$ we get

$$
\sum_{i=1}^{n} a_{i}(f) u_{i}^{(j-1)}\left(0, x^{*}\right)=f^{(j-1)}\left(0, x^{*}\right)
$$

for all $x^{*} \in B_{1}\left(X^{*}\right)$. Since equation (3) is valid for all $f \in C_{\omega}^{n}(I, X)$, it follows that for $x^{*} \neq 0, A_{n}\left(x^{*}\right)$ is non-singular. This ends the proof.

Lemma 3.2. Let $A_{n}\left(x^{*}\right)$ be non-singular, and $\left\|\sum_{i=1}^{n} a_{i}(\mathcal{E}) u_{i}\right\|_{I_{\mathcal{E}}}=0\left(\mathcal{E}^{n-1}\right)$ as $\mathcal{E} \rightarrow 0^{+}$. Then $a_{i}(\mathcal{E}) \rightarrow 0$ as $\mathcal{E} \rightarrow 0^{+}$for each $i=1, \cdots, n$. 
Proof. The proof follows from the facts

(i) $\left\|\sum_{i=1}^{n} a_{i}(\mathcal{E}) u_{i}\right\|_{I_{\mathcal{E}}}=0\left(\mathcal{E}^{n-1}\right)$ as $\mathcal{E} \rightarrow 0$ implies that $\left\|\sum_{i=1}^{n} a_{i}(\mathcal{E})\left\langle u_{i}, x^{*}\right\rangle\right\|_{I_{\mathcal{E}}}$ $=0\left(\mathcal{E}^{n-1}\right)$ as $\mathcal{E} \rightarrow 0$

(ii) If $g(t)=\left\langle u_{i}(t), x^{*}\right\rangle$, then $g^{\prime}(0)=u_{i}^{\prime}\left(0, x^{*}\right)$.

(iii) Lemma 2.1 of [4].

We now prove the converse of Theorem 3.1.

Theorem 3.3. Let $A_{n}\left(x^{*}\right)$ be non-singular for each $x^{*} \neq 0$ in $B_{1}\left(X^{*}\right)$. Then $P_{\varepsilon}(f)$ coverges uniformly to some $P_{0}(f)$. Further, $P_{0}(f)^{j}\left(0, x^{*}\right)=f^{j}(0$, $\left.x^{*}\right), j=1,2, \cdots, n-1$ and $x^{*} \in B_{1}\left(X^{*}\right)$.

Proof. Let $P_{\varepsilon}(f)=\sum_{i=1}^{n} a_{i}(\varepsilon, f) u_{i}$. For every $z^{*} \in B_{1}\left(X^{*}\right)$, the element $h=\sum_{i=1}^{n} f^{j-1}\left(0, x^{*}\right) u_{i}$ is an element of $M$. Since $P_{\varepsilon}(f)$ is the best approximant of $f_{\varepsilon}$ in $M_{\varepsilon}$, it follows that

$$
\left\|P_{\varepsilon}(f)-f_{\varepsilon}\right\| \leq\left\|h-f_{\varepsilon}\right\|_{I_{c}}
$$

Now, for any $g \in C(I, X)$, the map $t \rightarrow\|g(t)\|$ is continous on the compact set $I$. Hence $\sup _{t}\|g(t)\|=\left\|g\left(t_{0}\right)\right\|$ for some to. By the Hahn-Banach Theorem, there exists some $x^{*} \in B_{1}\left(X^{*}\right)$ such that

$$
\sup _{t}\|g(t)\|=\left\|g\left(t_{0}\right)\right\|=\left\langle g\left(t_{0}\right), x^{*}\right\rangle
$$

Consequently, since $h, f \in C(I, X)$, there exists some $t \in P_{\varepsilon}$ and $x^{*} \in B\left(X^{*}\right)$ such that

$$
\begin{aligned}
\left\|h-f_{\varepsilon}\right\| & =\left|\left\langle h(t)-f_{\bar{\varepsilon}}(t), x^{*}\right\rangle\right| \\
& =\left|\sum_{i=1}^{n} f^{(i-1)}(0, x)\left\langle u_{i}(t), x^{*}\right\rangle-\left\langle f_{\varepsilon}(t), x^{*}\right\rangle\right|
\end{aligned}
$$

Since $A_{n}\left(x^{*}\right)$ has an inverse, we can assume that

$$
\left\langle u_{i}^{(i-1)}(0), x^{*}\right\rangle=\delta_{i j}=\left\{\begin{array}{lll}
1 & \text { if } & i=j \\
0 & \text { if } & i \neq j
\end{array}\right\}
$$


Thus expanding each $\left\langle u_{i}, x^{*}\right\rangle$ arround the point $t=0$, using the Taylor expansion with remainder we get:

$$
\begin{aligned}
& \qquad \begin{aligned}
\left\langle u_{i}(s), x^{*}\right\rangle & =\left\langle u_{i}(0), x^{*}\right\rangle+\cdots+\frac{\left\langle u_{i}^{n-1}(0), x^{*}\right\rangle}{(n-1) !} s^{n-1}+\frac{\left\langle u_{i}^{(n)}(r), x^{*}\right\rangle}{n !} s^{n} \\
& =\left\langle u_{i}^{(i)}(0), x^{*}\right\rangle+R_{i}, \\
\text { where } \quad R_{i} & =\frac{\left\langle u_{i}^{(n)}(r), x^{*}\right\rangle}{n !} s^{n}, \quad 0<r<s<\varepsilon .
\end{aligned} \\
& \text { Hence } \sum_{i=1}^{n} f^{(i-1)}\left(0, x^{*}\right)\left\langle u_{i}(t), x^{*}\right\rangle=\sum_{i=1}^{n} f^{(i-1)}\left(0, x^{*}\right) \frac{t^{i}}{i !}+\sum_{i=1}^{n} R_{i} .
\end{aligned}
$$

Similarly, we expand $\left\langle f_{\varepsilon}(t), x^{*}\right\rangle$ around $t=0$, using Taylor series with remainder. Using the fact that $u_{i}$ and $f$ are in $C_{\omega}^{n}(I, X)$ for $i=1, \cdots, n$, and the fact each $R_{i}$ has the form

$$
R_{i}=\theta(r) \cdot S^{n}
$$

where $\theta$ is continous and $O<r<s<\varepsilon$, it follows that for $\varepsilon \rightarrow O^{+}$:

$$
\left|\sum_{i=1}^{n} f^{i-1}\left(o, x^{*}\right)\left\langle u_{i}(t), x^{*}\right\rangle-\left\langle f_{\varepsilon}(t), x^{*}\right\rangle\right|=O\left(\varepsilon^{n}\right)=o\left(\varepsilon^{n-1}\right)
$$

It follows from (1) that for $\varepsilon \rightarrow 0^{+}$:

$$
\left\|P_{\varepsilon}(f)-f_{\varepsilon}\right\|=o\left(\varepsilon^{n-1}\right)
$$

Now, let $b_{i}(\varepsilon, f)=a_{i}(\varepsilon, f)-f^{(i-1)}\left(o, x^{*}\right)$. Then

$$
\begin{aligned}
\left\|\sum_{i=1}^{n} b_{i}(\varepsilon, f) u_{i}\right\| & =\left\|\sum_{i=1}^{n} a_{i}(\varepsilon, f) u_{i}-\sum_{i=1}^{n} f^{(i-1)}\left(o, x^{*}\right) u_{i}\right\| \\
& \leq\left\|P_{\varepsilon}(f)-f_{\varepsilon}\right\|+\left\|\sum_{i=1}^{n} f^{(i-1)}\left(o, x^{*}\right) u_{i}-f_{\varepsilon}\right\| .
\end{aligned}
$$

Then using equation (2) and (3) to get for $\varepsilon \rightarrow O^{+}$:

$$
\left\|\sum_{i=1}^{n} b_{i}(\varepsilon, f) u_{i}\right\|=o\left(\varepsilon^{n-1}\right)
$$


Hence,

$$
\lim _{\varepsilon \rightarrow 0^{+}}\left\|P_{\varepsilon}(f)-\sum_{i=1}^{n} f^{(i-1)}\left(o, x^{*}\right) u_{i}\right\|_{I_{\mathcal{E}}}=0
$$

Thus $P_{\varepsilon}(f)$ converges uniformly to some $P_{0}(f)$ as $\varepsilon \rightarrow O^{+}$. Further, equation (4) and Lemma 3.2 implies that $\lim _{\varepsilon \rightarrow 0^{+}} b_{i}(\varepsilon, f)=0$. Hence

$$
\lim _{\varepsilon \rightarrow O^{+}} a_{i}(\varepsilon, f)=f^{i-1}\left(o, x^{*}\right)
$$

Thus

$$
P_{0}(f)^{j}\left(o, x^{*}\right)=f^{j}\left(o, x^{*}\right)
$$

This ends the proof of the Theorem.

Closing Remarks. One can consider the problem of best local approximation for different subspace $M$. Indeed Let $X=\ell^{p}, 1 \leq p<\infty$, and $L^{p}\left(I, \ell^{p}\right)$ be the space of $p$-Bachner integrable functions defined on $I$ with values in $\ell^{p}$. Hence for $f \in L^{p}\left(I, \ell^{p}\right)$,

$$
\|f\|_{p}=\left(\int_{0}^{1}\|f(t)\|^{P} d t\right)^{1 / p}=\left(\int_{0}^{1} \sum_{n=1}^{\infty}\left|f_{n}(t)\right|^{P} d t\right)^{1 / p}
$$

where $f(t)=\left(f_{n}(t)\right)_{n=1}^{\infty}$.

Let $\left\{u_{i}, \ldots, u_{n}\right\}$ be continuous functions in $L^{P}\left(I, \ell^{P}\right)$ such that

$$
u_{j}=\left(u_{j i}\right)_{i=1}^{\infty},
$$

and $\left\{u_{1 i}, \ldots, u_{n i}\right\}$ is a $T$-system in $C(I)$ for each $i=1,2,3, \cdots$. Set $M=$ span of $\left\{u_{i}, \ldots, u_{n}\right\}$. In [6] Kroo proved that $M$ is a Chebechev subspace in $L^{1}\left(\ell_{n}^{2}\right)$, $\ell_{n}^{2}$ is a finite dimensional Hilbert space. The authors proved in [1] that $M$ is a Chebechev subspace in $L^{1}\left(\ell^{p}\right)$, for any $1 \leq p \leq \infty$, and with no restriction on the dimension of $\ell^{p}$. If we set $p=1$ and $M_{j}=\operatorname{span}\left\{u_{11}, \ldots, u_{n j}\right\}$, then each $M_{j}$ is a Chebechev subspace in $L^{1}(I)$ by the Jackson's Theorem [3]. Set

$$
M=\left\{\left(g_{j}\right): g_{j} \in M_{j}: \int_{0}^{1}\|g(t)\| d t<\infty\right\} .
$$


Then $M$ is a closed suispace of $L^{1}\left(I, \ell^{1}\right)$. Further $M$ is proximinal. For $f=$ $\left(f_{n}\right) \in L^{1}\left(I, \ell^{1}\right)$ and $\hat{f}_{n} \in M_{n}$ such $d\left(f_{n}, M_{n}\right)=\left\|f_{n}-\hat{f}_{n}\right\|_{1}$, we have $g=\left(\hat{f}_{n}\right) \in$ $M$ and

$$
d(f, M)=\|f-g\| .
$$

In this case the problem of best local approximation is that for the coordinate functions $f_{n}$, and one can prove

Theorem. $P_{\mathcal{E}}(f)$ converges in $L^{1}\left(I, \ell^{1}\right)$ to some $P_{0}(f)$ in $M$ if only if $P_{\mathcal{E}}\left(f_{n}\right)$ converges in $L^{1}(I)$ to some $P_{0}\left(f_{n}\right)$ in $M_{n}$.

\section{References}

[1] A. Al-Zamel, and R. Khalil, "Unicity spaces in vector valued function spaces." Submitted. (1989).

[2] J. Diestel, and J. Uhl, "Vector measures," Math. Surveys, no. 15 (1977).

[3] W. Cheney, "Introduction to approximation theory," McGraw-Hill comp. New York. (1966).

[4] C. Chui, O. Shisha, and P. Smith, "Best local approximation," J. of approx. 15 (1975), 371-381.

[5] W. Light, and E. Cheney, "Approximation theory in tensor product spaces," Lecture notes in Math 1169. (1985).

[6] A. Kroo, "Best $L^{1}$-approximation of vector valued functions," Acta Math. Acad. Sci. Hungar., 39 (1982), 303-313,

Kuwait Univ., P O Box 5969, Kuwait.

Bahrain Univ., Kuwait P O Box, Isa Town Bahrain. 